\title{
Population PK and Dosing of Milrinone After PDA Ligation in Preterm Infants
}

Maarja Hallik ${ }^{1,2}$, Mari-Liis IImoja², Tõnis Tasa ${ }^{3}$, Joseph F Standing ${ }^{4}$, Kalev Takkis ${ }^{5}$, Rūta Veigure ${ }^{6}$, Karin Kipper ${ }^{5,6}$, Tiiu Jalas²; Maila

Raidmäe ${ }^{2}$, Karin Uibo², Joel Starkopf ${ }^{1,7}$, Tuuli Metsvaht ${ }^{7,8}$

1 Department of Anesthesiology and Intensive Care, Institute of Clinical Medicine, University of Tartu, Tartu, Estonia; 2 Clinic of Paediatrics, Tallinn Children's Hospital, Tallinn, Estonia; 3 Institute of Computer Science, University of Tartu, Tartu, Estonia; 4 Inflammation, Infection and Rheumatology section, Great Ormond Street Institute of Child Health, University College London, London, United Kingdom; 5 Analytical Services International, St George's University of London, London, United Kingdom; 6 Institute of Chemistry, University of Tartu, Tartu, Estonia; 7 Clinic of Anaesthesiology and Intensive Care, Tartu University Hospital, Tartu, Estonia; 8 Department of Pediatrics, Institute of Clinical

Medicine, University of Tartu, Tartu, Estonia

\section{Background and aims:}

- The postoperative course of PDA ligation is often complicated by post ligation cardiac syndrome (PLCS), occurring in $10-45 \%$ of operated infants.

- Milrinone has been used to prevent profound hemodynamic instability in this setting, but the efficacy depends on optimal dosing.

- The present study aimed to describe the population PK of milrinone in premature neonates at risk of PLCS and give dosing recommendations.

\section{Methods:}

- A prospective two-centre study was performed in Tallinn Children's Hospital and Tartu University Hospital, Estonia.

- Milrinone was administered to infants at risk of PLCS, reflected by left ventricular output of less than $200 \mathrm{ml} / \mathrm{kg} / \mathrm{min}$ 1 hour after the PDA surgery, patients with renal failure or major congenital malformation were excluded.

- The dose of $0.73 \mu \mathrm{g} / \mathrm{kg} / \mathrm{min}$ for 3 hours followed by $0.16 \mu \mathrm{g} /$ $\mathrm{kg} / \mathrm{min}$ for 21 hours was attained by a prior simulation study with the aim of reaching therapeutic plasma concentrations in 3 hours. *

- Concentration-time data were analysed with nonlinear mixedeffects software (NONMEM version 7.3).

- Probability of target attainment (PTA) simulations were aimed at dosing schedule that maximally attains concentration targets at $3,6,12,18$ and 24 hours of treatment.
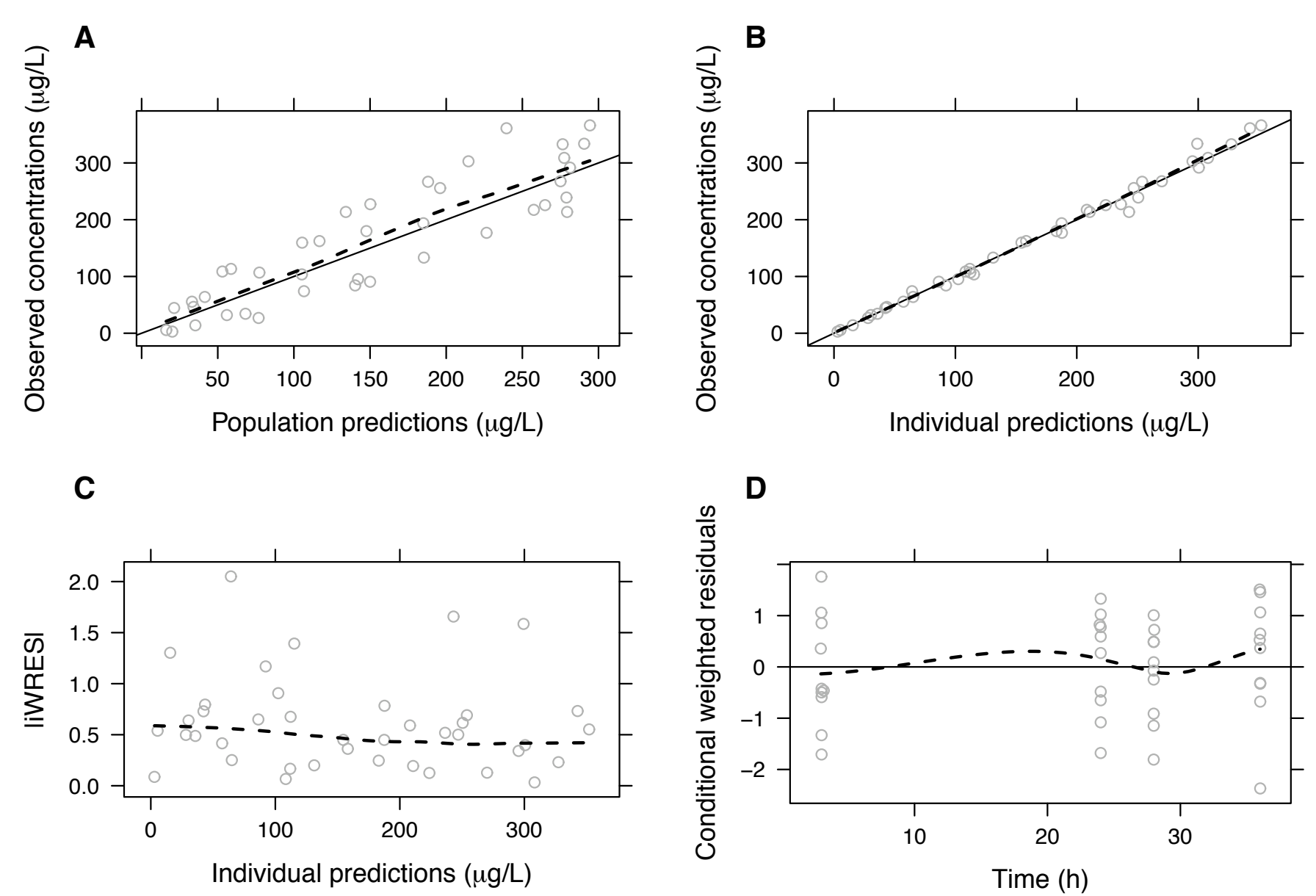

Figure 2. Optimization results of loading and maintenance phase doses.

\section{Results:}

- Among 21 operated neonates 14 were included, 10 of them were treated with milrinone.

- Mean (range) PMA 27.4 (24.6 - 30.1) weeks, PNA 16 (5 $27)$ days and weight $857.3(568-1114) \mathrm{g}$.

- A total of 40 milrinone plasma concentrations were collected.

- Milrinone PK was described by one-compartmental linear model with allometric scaling to bodyweight and maturation function of GFR ${ }^{* *}$ and proportional residual error. (Figure 1)

$$
\begin{aligned}
& C L=\theta_{C L} \cdot\left(\frac{W D 1}{857.3}\right)^{0.632} \cdot \frac{P M A^{3.4}}{47.7^{3.4}+P M A^{3.4}} \\
& V d=\theta_{V d} \cdot\left(\frac{W D 1}{857.3}\right)^{1}
\end{aligned}
$$

- Population mean (CV\%) parameter estimates were 0.350 (33\%) L/h for clearance and $0.329(12 \%)$ L for volume of distribution.

- PTA simulations suggest a loading dose of $0.50 \mu \mathrm{g} / \mathrm{kg} / \mathrm{min}$ for 3 hours followed by $0.15 \mu \mathrm{g} / \mathrm{kg} / \mathrm{min}(\mathrm{PMA}<27$ ) or $0.20 \mu \mathrm{g} /$ $\mathrm{kg} / \mathrm{min}(\mathrm{PMA} \geq 27)$ to attain milrinone plasma concentrations of 150-250 $\mu \mathrm{g} / \mathrm{L}$. (Figure 2)

\section{Conclusion:}

PK of milrinone in preterm infants is affected by rapid changes in body water content and renal function maturation. Thereof PK parameters in our study population differ significantly from earlier described in the population of preterm neonates within the first 3 days of life. ${ }^{* *}$
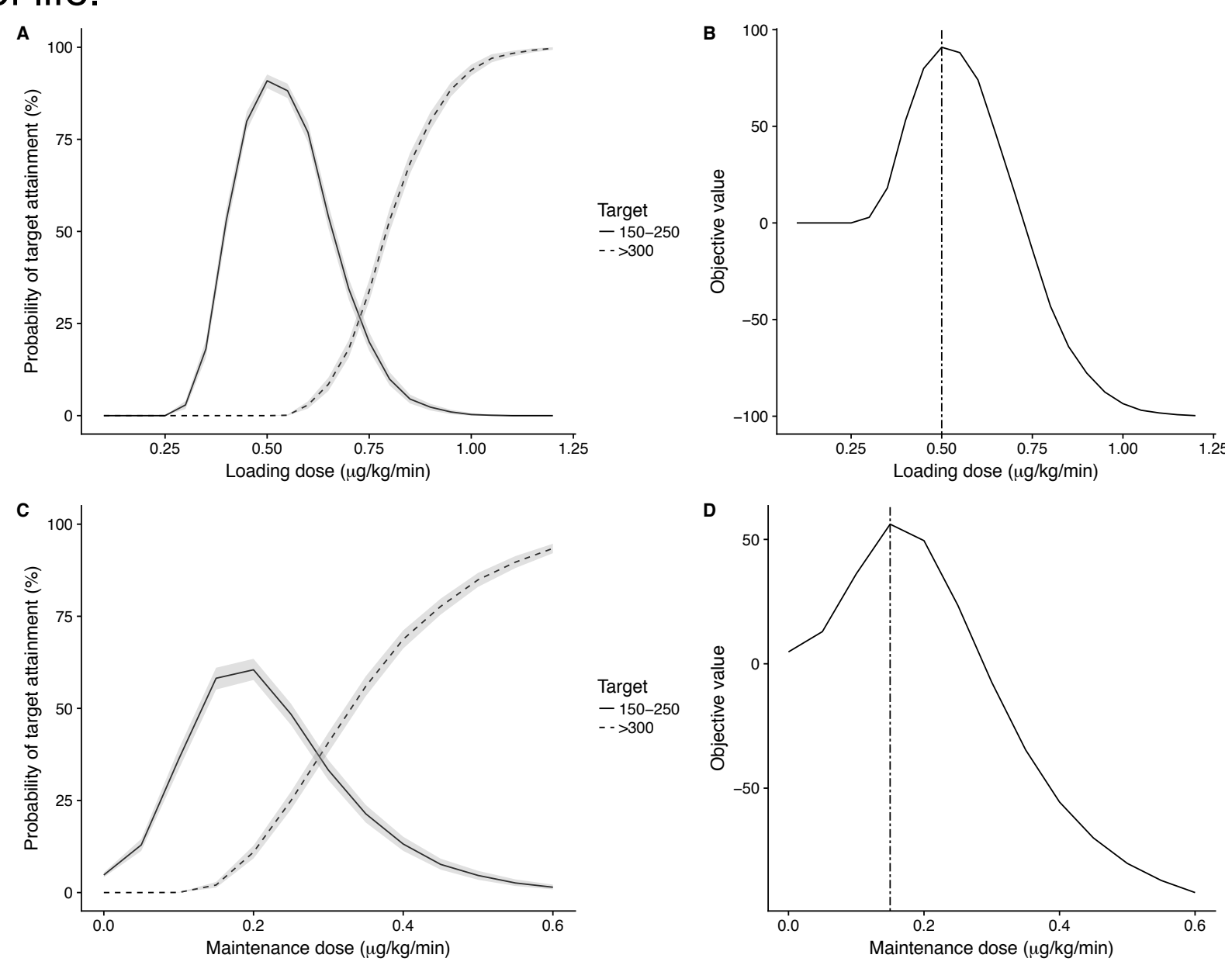

Figure 1. Basic goodness-of-fit plots of the final PK model.

* Hallik M, Tasa T, Starkopf J, et al: Dosing of Milrinone in Preterm Neonates to Prevent Postligation Cardiac Syndrome: Simulation Study Suggests Need for Bolus Infusion. Neonatology 2017; 111(1):8-11

** Rhodin MM, Anderson BJ, Peters AM, et al: Human renal function maturation: a quantitative description using weight and postmenstrual age. Pediatr Nephrol 2009; 24(1):67-76

${ }^{* * *}$ Paradisis M, Jiang X, McLachlan AJ, et al: Population pharmacokinetics and dosing regimen design of milrinone in preterm infants. Arch 